\title{
Family Storytelling for Grandparents and Grandchildren living apart
}

\author{
René Vutborg ${ }^{1}$, Jesper Kjeldskov ${ }^{1}$, Sonja Pedell ${ }^{2}$ and Frank Vetere ${ }^{2}$ \\ ${ }^{1}$ Aalborg University, Department of Computer Science, Denmark, rene@vutborg.dk, jesper@cs.aau.dk \\ ${ }^{2}$ The University of Melbourne, Australia, pedells@unimelb.edu.au, f.vetere@unimelb.edu.au
}

\begin{abstract}
Grandparents may feel revitalized when a grandchild joins the family, but the physical separation that often exists between grandparents and grandchildren can make it difficult to develop a close relationship. Current communication technologies, such as the phone, are inadequate for developing close relationships with children. This paper presents the design, implementation and evaluation of a technology probe exploring how technology can be designed to alleviate this problem. Based on the evaluation, four important themes for designing technology for distributed intergenerational bonding are elicited and discussed. The four themes are Conversational Context (to have something to talk about), Facilitation (to be given the opportunity to talk), Diversified Interaction Forms (to maintain attention of the child) and Supporting Grandparent caring for grandchild (to adapt activity to the mood of the child).
\end{abstract}

\section{Author Keywords}

Conversational context, grandchildren, grandparents, bonding, distributed interaction, field study, technology probe

\section{ACM Classification Keywords}

H5.m. Information interfaces and presentation (e.g., HCI): Miscellaneous.

\section{INTRODUCTION}

The majority of urban households comprise of no more than two generations [18]; one or two adults, who might be parent(s) for one or more children. The older generation, the grandparents, typically lives in their own house. This physical separation between grandparents and their grandchildren almost inevitably makes it harder to develop and maintain a close relationship between the two. Social circumstances such as parents getting divorced can further exacerbate these opportunities. Studies show that a close relationship is important for the well being of both grandparents and grandchildren [8,9]. The geographical separation and the inadequacy of current communication technologies

Permission to make digital or hard copies of all or part of this work for personal or classroom use is granted without fee provided that copies are not made or distributed for profit or commercial advantage and that copies bear this notice and the full citation on the first page. To copy otherwise, or republish, to post on servers or to redistribute to lists, requires prior specific permission and/or a fee.

NordiCHI 2010, October 16-20, 2010, Reykjavik, Iceland.

Copyright 2010 ACM ISBN: 978-1-60558-934-3...\$5.00. (telephone, webcam) pose a threat to the development of this relationship. Grandparents, or other distant relatives, find it hard to get the child engaged in a conversation over the phone [2]. This is the case for most children until they reach 7 or 8 years of age [2]. The child is often bored when talking on the phone and would much rather roam the house or play with friends [1].

Collocated talk between grandparents and grandchildren is often submerged in ongoing activities [5]. By mediating the normally collocated activity of storytelling, we provided geographically separated grandparents and grandchildren with shared activities during sessions of synchronous contact. The goal was to explore if children, under the age of 8 years, can be made interested in engaging with their grandparents if such activities are provided.

First, we present related work concerning the importance of a good grandparent/grandchildren relationship. This is followed by an overview of previous research on using technology to facilitate contact between distributed grandparents and grandchildren. The process of designing, implementing and deploying the technology probe is then presented. Finally, findings from the analysis are presented and discussed by eliciting themes for designing technology to support distributed intergenerational bonding.

\section{RELATED WORK}

Kornhaber [8, 9] conducted a 3-year sociological study involving 300 sets of grandparents and grandchildren and found the grandparent-grandchild relationship to be important for both. They found the grandparent-grandchild bond to be of second most emotional importance to the very close parent-child bond [9] and that "Grandparenting provided many elders with meaning and joy in their lives" [8]. For the grandchildren, a good relationship with their grandparents makes them feel emotionally secure, having them as a "backup" if their parents fall away and provides them almost unconditional love [8]. Kornhaber also found that shared grandparent/grandchild activities build up the child's self-esteem [8]. One potential way such relationship can arise is through shared collocated activities. Kennedy [7] asked 391 young adult grandchildren to write down which shared activities they had with their grandparents. Based on more than 1000 nominations for types of activities, Kennedy generated 29 categories for different types of grandparent-grandchild shared activity. Examples of such catego- 
ries are "attending church together" and "spending the night at grandparent's house".

Current synchronous mainstream technologies that provide contact opportunities for geographically distributed persons use an audio channel or a combined audio and video channel, but these technologies all pose challenges for successful bonding when a child is involved. Ballagas et al. found that "young children [...] have difficulties articulating clearly with words alone" and that "children up to 9 years old had difficulties staying engaged in the phone conversation" [2]. Ames et al. found that when children were participating in video chat sessions with remote family members, all children had a hard time sitting still in front of the camera. They would much rather roam around the house, thus making the family members at the other end of the video chat unable to see and hear the child [1].

Several projects have, in various ways, tried to stimulate children's interest in synchronously communicating with remote relatives using technology. Yarosh et al. [19] built the ShareTable system, aimed at parent-child contact, where a combination of a camera, a projector and special projection surface allowed the child and parent to share viewing of physical artefacts. Evaluation showed that the ShareTable system was well received by both parents and children and preferred over regular videoconferencing, although some children had a hard time understanding how the system worked. Vetere et al. [17] made the Collage system for exploring intergenerational distributed play. The grandparents and grandchildren could send photos and text messages from a mobile phone to the system, and both could then manipulate these objects on each their touch screen monitor. Manipulation would be synchronously replicated to the other household. Evaluation showed that both grandchildren and grandparents enjoyed the new types of playful activities the system offered. Modlitba [11] developed the GlobeToddler system making the child able to synchronously communicate with a travelling parent. The parent can record audio comments and take pictures and send them to the child (asynchronously), who is supplied with an interactive doll based on a Nintendo Wii remote controller. Whenever the child uses the doll, the parent is notified of this, and can choose to engage in a synchronous activity with the child, for example, by making an avatar jump. Evaluation showed that both parent and child really enjoyed the system, but it also showed that the child sometimes found the doll confusing to use and not consistent enough. Raffle et al. [12] created a custom-made device to be used by grandparents and grandchildren for shared reading of physical storybooks. It included an audio channel and page sensing technology to determine if the child was on the same physical page. Evaluation showed that it made children more engaged in long-distance communication than when they used Skype and that the quality of the intergenerational interactions improved as well.

This paper reports research exploring intergenerational interactions over distance. We present the design, develop- ment and evaluation of an investigative prototype deployed as a "technology probe" [6] in four households: two with grandparents and two with grandchildren.

\section{RESEARCH DESIGN}

Previous research has explored how to facilitate synchronous contact between family members living apart by introducing custom-made technology into homes and by interviewing their inhabitants $[11,19,17]$. This paper is the outcome of a similar approach, where a technology probe [6] with data logging capabilities was deployed to families and where the family members were interviewed before and after they had used the system provided. The pre-use interview focussed on challenges of maintaining contact with grandchildren living apart. The deployed system explored ways of supporting distributed intergenerational contact by mediating an otherwise typically collocated shared activity. The post-use interview focused on how the system facilitated distributed contact.

\section{Case}

Grandparents and grandchildren participate in various types of activities when together [7]. The shared activity storytelling was selected because it seemed feasible to mediate over distance compared to, for example Kennedy's category of "eating out with grandparent" [7]. Storytelling is in the context of this paper defined as the oral activity of telling, that is, conveying a story from one to another. In the context of this paper a story is defined as either real, for example about daily life, corresponding to Kennedy's category of "Talking together about recent events in each other's lives" or fictional and read out loud, corresponding to Kennedy's category of "Reading books and telling stories together". The goal is to mediate both telling of real and fictional stories.

\section{THE STORYTELLING SYSTEM}

The goal of the storytelling system is to explore if grandparents and grandchildren find it fun, meaningful and possible to share activity over distance and, if this is the case, how they choose to do it. The system had to require no intervention from researchers during use, as this would potentially be annoying for the participating families. The age of the participating family members also had to be considered. It was important that children and grandparents did not find the system boring and that they were not intimidated by it. Even though the technology probe presented in this section can be seen as a prototype, the goal is to explore how technology can be used to facilitate contact between distributed grandparents and grandchildren than exploring technical or usability related matters.

\section{Conceptual Design}

The conceptual design of the storytelling system was inspired by Yarosh, who built the ShareTable for shared viewing of physical objects [19]. 


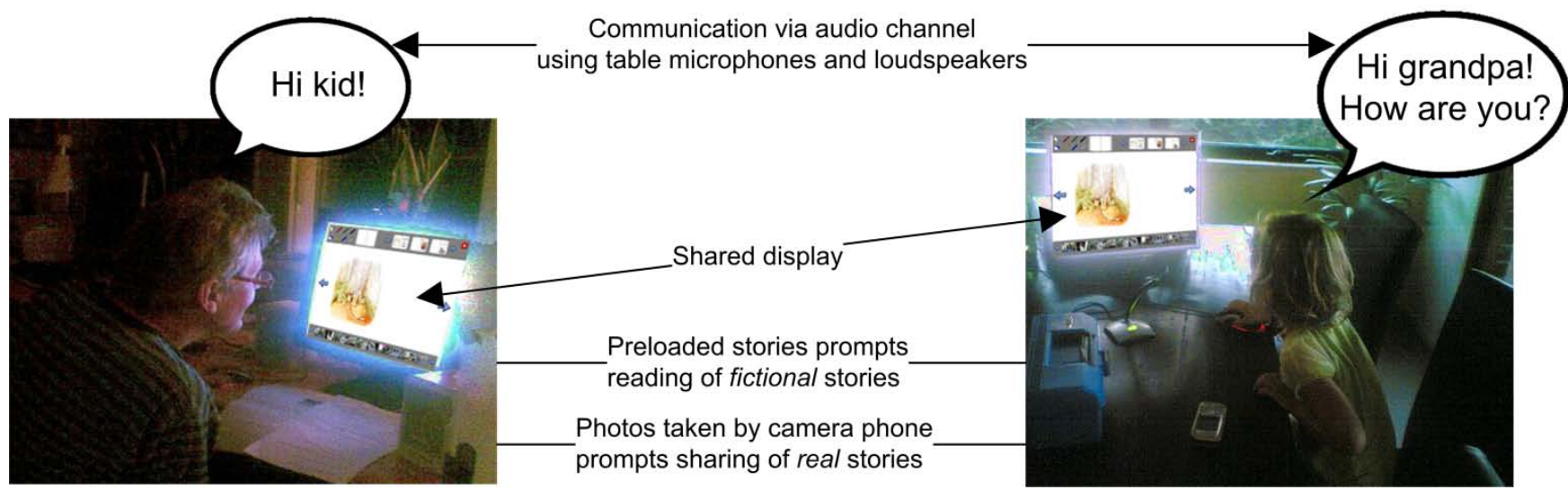

Figure 1: The basic design concept for the distributed storytelling system.

Adapting this to a less physical context, but with the same objective - shared viewing - the technology probe was designed to allow sharing of virtual objects, through a shared display. Thus the system was made to consist of two LCD monitors and two computers, one set for each household. The shared display was implemented by replicating one household's interaction with the probe on the monitor in the other household and vice versa, thus following the WYSIWIS (What You See Is What I See)-concept [14]. The technology probe was furthermore equipped with an audio channel through which grandparents and grandchildren could talk to each other as on a normal phone. An important part of bonding is developing trust in each other and research shows that "speech plays a significant role in that development" [5]. Research also shows that trust indeed can develop in a non face-to-face situation [3]. Thus, as the motivation behind the research grounding this paper is to facilitate bonding between grandparents and grandchildren living apart, an audio channel was deemed necessary. Supplying the system with an audio channel also conveniently solves the potential issue of asking children in the early stages of learning how to read and write to communicate using a text-only medium. Figure 1 illustrates the basic design concept.

Making it a technology probe: "an instrument that is deployed to find out about the unknown" [6] the storytelling system was equipped with recording capabilities for making all audio communication and visual interaction available for subsequent analysis by the researchers.

Furthermore, the system was built of generally available hardware and software technologies, combined in a novel way. Each household was equipped with a table-based microphone and a set of loudspeakers to allow an audiochannel to be opened. This approach was preferred over providing headsets as it would allow multiple people to talk at once in both households, and as children would not have to put on headsets before audio contact could be made.

\section{Interface and Interaction Design}

The visual interface made available to the households comprises two parts: initiation of storytelling sessions and ac- tive storytelling sessions. One household can at any time choose to invite the other to participate in a storytelling session, and the other household then have the option to either accept or decline the invitation. Whenever one household invites the other, a single telephone sound is played in both households to draw attention to the system. Upon acceptance of the invitation, the interface for active storytelling sessions is shown. Both households can close the session at any time by clicking on the red $\mathrm{x}$ in the top right corner. To keep the system simple, and to allow grandparents and grandchildren to interact around something shared, the visual interface is identical in the two households. This interface is illustrated in Figure 2. Supplying both households with the same interface to make it easier for them to help each other is previously shown to be effective. Kraut et al. found that sharing the visual space helps establish common ground between two distributed persons when the objective is for one to help the other [10].

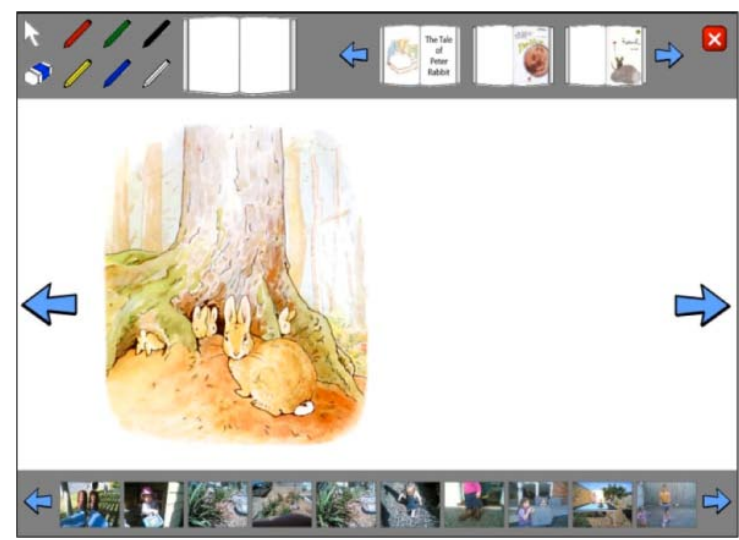

Figure 2: The interface design of an active storytelling session, where the first page from the fictional book "Peter Rabbit" is chosen. The large white area in the middle is the shared space. The bottom row is the photo toolbar. The top row toolbar contains the books and the coloured pencils.

The interface for active storytelling sessions contains two conceptually different parts. One part is a shared visual space [10] where everything shown, and all actions done, is replicated to the other household, hence in effect making it a shared space. Having such a shared space is shown to 
improve communication between distributed people [10]. The other part is toolbars, where the different mediated storytelling activities can be initiated. This distinction can be seen on Figure 2. The shared visual space was designed with an unlimited amount of empty slides (as in, for example, PowerPoint), which both households can navigate between freely using the left and right arrows. The following describes the different types of visual elements available in the system for inserting onto the slides.

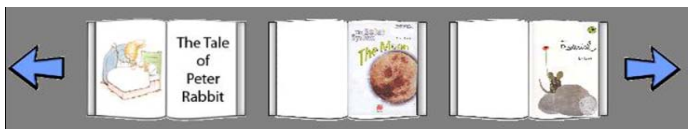

Figure 3: Using the arrows different storybooks can be read.

The first type of mediated activity available facilitates fictional stories. When a fictional book is read aloud in a collocated setting, both the grandparent and grandchild can touch the book, see the book and influence the choice of what book to read. This was mediated in the system by making 10 different children's books available in the toolbar section, as seen in Figure 3. Both grandparents and grandchildren are given the opportunity to select what book to read and to navigate the pages of the book by pressing the left- and right-pointing arrows. When a book from the toolbar is selected in one household, the same book is shown on the display in the other household. The same is the case when navigating pages. The hope was that these shared books would make grandparents and grandchildren interested in reading aloud stories to each other.

The second type of mediated activity available facilitates exchange of real stories in relation to questions like "what have you been doing today?". With the purpose of making conversation around those types of questions easier, the system was designed with the possibility to easily share photos. This was done by supplying a designated camera phone for each household to use at their own discretion, whether in the house or on the move, and then share these photos through the system. It was hoped that these photos would make it easier for especially children to remember and prompt stories about their day. Figure 4 illustrates how these photos are made available in the bottom toolbar section. Both households have the possibility to drag a photo from this toolbar into the shared visual space. When a photo is uploaded from one household's mobile phone, it is automatically placed in the photo toolbar in both households. The idea behind this decision was to make a scenario possible where the grandparent would drag a photo taken by the grandchild into the shared visual space and then ask questions along the line of "what are you doing here?" thus prompting conversation. An unlimited amount of photos can be inserted on the same slide, photos can be moved around and photos can be resized with or without maintaining proportions. Supplying the grandparents and grandchildren with the possibility to share photos and read children's books is well in line with previous research concluding that interfaces for children "should [...] elicit sharing or storytelling" [2].

A series of coloured pencils and an eraser were also added to the toolbar section. These pencils can be used to draw on top of storybooks, photos and blank slides and the eraser can be used to erase it again. As every parent knows, children love to paint. It was hoped that children would use these pencils to perhaps make paintings for the grandparent or that grandparents would use these pencils to tease the grandchild, a trend Ballagas et al. found grandparents would use to "engage the children in the conversation" [2]. Ballagas et al. also found that children are in general more inclined to express their ideas through action than through words [2], a finding the pencils hopefully support well. Finally, touch-enabled LCD monitors were used. This allowed children to paint with the pencils on the monitor with their fingers. It also allowed more than one person to interact with the system simultaneously without having to fight over control of the mouse. However, the mouse was still available too, if needed.

\section{Technical Design}

The system and probe software was implemented in $1 \frac{1}{2-}$ month time by a single researcher. The audio channel between the two households was implemented using an existing piece of audio communication software [16]. The interface displayed on Figure 2 was implemented in a C\# based Windows Forms client. It communicates with a Microsoft SQL Server database in real-time to ensure that actions from both households are replicated in the other household. Finally, photos are taken with a Nokia 7610 camera phone, and via MMS sent to an e-mail address. A C\# based console application then extracts these photos from the e-mail address at regular intervals, saves them in the SQL Server database and adds the photos to the photo toolbar in both households. The technical design is illustrated on Figure 5.

\section{DEPLOYMENT}

The technology probe was deployed in a field study with two participating families. The objective was to evaluate how the grandparents and grandchildren used the system to facilitate and augment their interpersonal contact and communication. The families received no remuneration but had the costs of their involvement covered.

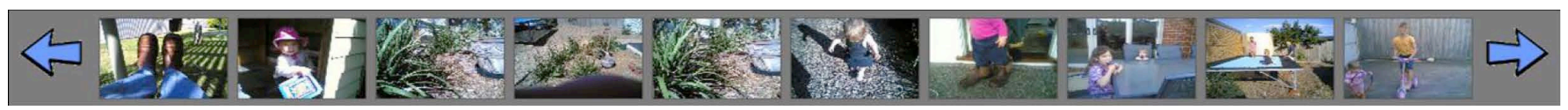

Figure 4: The photo toolbar. Using the left and right arrows all photos can be seen and inserted. 


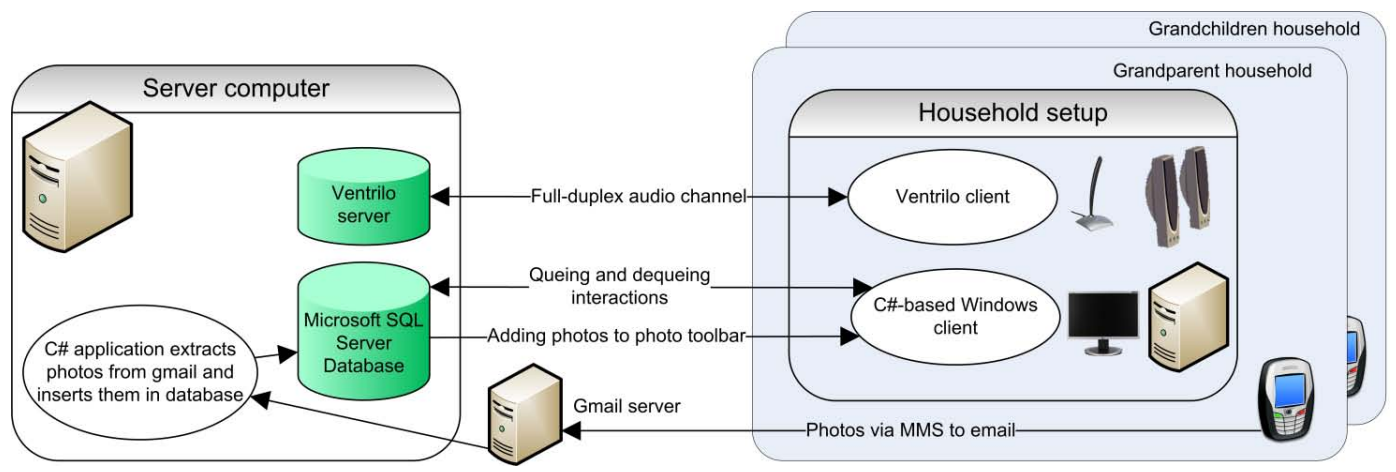

Figure 5: Technical structure of the system.

\section{Participants}

The participating families were recruited through academic staff at the University of Melbourne, Australia. Each family had to meet a basic set of requirements to be selected for participation. Both households in the family had to be located in the greater Melbourne area. The parents had to have at least one child aged between 4 and 8 , and the grandparents had to live in their own household and require no external care. These requirements were put in place to ensure that the family would, and could, invest the time required to generate useful data. The participating families had complete freedom regarding frequency, content and time of use. Several families volunteered to participate in the study among which the following two families were chosen. Family 1 consisted of two children aged 5 and 6 and two grandparents. They normally kept in contact using the phone and lived around $30 \mathrm{~km}$. from each other. Recent shared activities between the grandparents and grandchildren included the children having sleepovers at the grandparent household, baking, and going to science museums. Family 2 consisted of three children aged 2, 6 and 8 and two grandparents. They also normally used the phone to keep in touch, and lived around $20 \mathrm{~km}$. from each other. Recent shared activities include looking at books together, reading stories and painting.

\section{Method and Data Collection}

When a family had agreed to participate in the study, identical set up dates were scheduled with both households to minimize the amount of time one household would have a non-working probe. At the scheduled date, two researchers drove to the two households. The following procedure was then followed for both households in both families.

First, a semi-structured interview was conducted. The intention of this interview was both to "break the ice" between the household residents and the researchers, but more importantly to achieve a basic understanding of how that household communicated with the other household. Among the questions asked were how they currently stay in contact with the other household and which problems they have experienced with this. Secondly, the probe computer and the 19-inch touch screen display were installed in the household. To facilitate access and use, the touch screen was placed in a central and high traffic area of the house, such as on a kitchen table. The computer and the keyboard were hidden away as much as possible. The mouse was kept visible and usable as a supplement to the touch screen. The computer was wirelessly connected to the household's existing ADSL-based Internet connection (all households had that already). The family was then informed that they could use the system in whatever way they found suitable or exciting. After having briefly demonstrating the available toolbars and functionality, ensured that the audio channel worked, and instructed the household's residents on how to send photos from the camera phone, the two researchers left. While the probe was deployed at the families it was monitored remotely and technical problems were solved as quickly as possible. Every time the family used the system, a screen capture program [4] recorded both what was shown on the screen and their voice talk. This happened automatically in the background without interfering with the use of the system. This was supplemented by a log file with time stamped entries of every time a household tried to initiate a session, what the response was from the other household ("accept" or "decline") and how long sessions lasted. When the probe was collected from the households again, a second semi-structured interview was conducted investigating how they used the system, what they liked and disliked and which tools they used the most. During both the pre- and post-use interview, the most valuable comments came from the grandparents and parents, as the grandchildren generally had a hard time maintaining focus on the interview.

The probe had to be deployed sequentially to the two families for technical reasons. To gather enough data and ensure that system usage routines developed, the intended duration of deployment in a family was four weeks. However, family 1 had the probe for only 14 days as they chose to opt out. This was caused by lack of time to use the system in the grandchildren household. They used it for only $30 \mathrm{~min}-$ utes in total. To compensate this the probe was deployed for an extended period of 45 days at family 2 . This family used the system for a total of 5 hours and 3 minutes.

\section{Data Analysis}

Even though the storytelling activity was what the system was mainly designed to facilitate, it would limit the analysis if storytelling activities were the only area of focus because 
the nature of a technology probe is to explore the unknown, and because the participants can "use them [the probed systems] in unexpected ways" [6]. Vetere et al. observed grandchildren and grandparents together in collocated playgroups and derived categories for shared collocated play [17]. Among these categories are instruction (e.g. how to use an object), performance (e.g. singing a song), game (e.g. playing with a ball) and joking (e.g. telling an obvious lie). Besides the storytelling activity, these four categories further worked as inspiration for types of shared activities to look for in the analysis. Using a Grounded Theory approach [15], inductive knowledge was created in the following systematic way. The video recordings of the storytelling sessions, approximately $5 \frac{1}{2}$ hours in total, were transcribed and structured into columns. The first column contained a direct transcription of the speech. The second column contained a description of what they did in the shared space (e.g. "Grandparent inserted a photo"). During this process, whenever an interesting event occurred, this was noted in a third column, representing the properties in Open Coding. A total of 238 properties were identified, which were subsequently categorized as 47 different phenomena. Using Axial Coding, connections were made between these phenomena, resulting in 12 categories. Using Selective Coding, these categories were divided among three themes, each of which is central to the use of the probe and each of which is treated in the next section. This process took 31 hours to complete.

\section{FINDINGS}

The analysis revealed a few issues with the basic design setup, several instances of successful mediation of collocated shared activity and even instances where existing types of activity were mediated in a novel way. A common observed issue was that the system lacked information supplying the grandparent with information about what the grandchild was doing. The grandparent would ask, as an example, "Which photo are you talking about", and the grandchild would then point on the particular photo and say "this one!". As the system did not indicate to the grandparent where the grandchild was pointing, the grandparent had no way of knowing what photo the grandchildren were referring to. This happened several times and suggests that the child did not understand what actions grandparent can and cannot see. This issue could be addressed by, for example, displaying the current position of the grandchild cursor on the grandparent monitor. However, this could also reduce playfulness without really adding functionality that wasn't already there, as another observation revealed where a child figured out how to use one of the coloured pencils as a pointer by painting on top of the photo in question.

Despite these issues, the video recordings from both families showed that both grandparents and grandchildren had a really good time together via the system.

\section{Observed activities}

A total of 17 sessions were conducted. The average length of a session was 14 minutes with a maximum length of 33 minutes. The grandchildren household were most eager to initiate sessions (59\%), however an indeterminable amount of these attempts were, in fact, sessions initiated by the mother rather than the grandchildren.

\section{Reading stories}

Reading the fictional stories aloud was a popular activity among grandparents and grandchildren. Most often, the grandchild chose the story, which the grandparent then read to the grandchild, adapting his or her voice to the story character. Sometimes the grandchild got really into the story, and did nothing else than turning the page of the book when instructed to by the grandparent. The longest session with a grandchild $100 \%$ immersed into the story lasted close to 17 minutes. This is very similar to the way one of the grandparents reported $\mathrm{s} / \mathrm{he}$ told stories with collocated grandchildren by sitting down with them and reading a book. Beside these sessions, where the grandchild said nothing for a long period of time, the storybooks often prompted conversation. The grandparents often explained the meaning of words and asked questions about the story to the grandchild, who eagerly answered. The grandparents also related the story to the real world, e.g. by elaborating on a story item (e.g. explaining where Eucalyptus trees grow because a Eucalyptus tree was present in a story). This is very similar to other activities that the grandparents reported doing with their grandchildren when physically together. Other times, the grandchildren were really active during the grandparent's reading out loud. A common behaviour by the grandchildren was to paint on top of story characters, which amused both parties. In one episode, a grandchild painted red spots on top of a story character, which prompted the grandmother to say "Ohh, he's got measles", which made the child laugh. Some stories also made the grandparent and grandchild act out specific lines from the story by yelling the line into the microphone, which also amused both parties. The grandparents also often suggested that the child read a book out loud. The child agreed to do this only a few times, and the child's reading aloud lasted only a few pages before the child lost concentration or asked the grandparent to read instead. During these attempts of the child to read aloud, the grandparent helped with the reading by pronouncing difficult words to the child, who then repeated the word.

\section{Sharing photos}

The photos taken with the camera phone turned out to provide opportunities for talk as well. During the time where the probe was deployed in the families, family 1 and family 2 took and sent 17 and 77 photos, respectively. In one episode, a photo of a papier mâché crocodile made by the grandchild was shared, and the grandchild eagerly answered all the grandparent's questions about their creation. Other photos induced similar patterns of talk. The ability to change the proportion of photos also proved to trigger laughter. In one example, the granddaughter inserted a photo of her older brother, and said "Then I make it [the photo] biiig [taller] and then I squash him [minimize width 
dramatically]". This caused the grandparent to reply "What does that feel like, squashing your brother? Does that feel good?" which the grandchild answered with loud laughter and a "yes". The coloured pencils were also often used combined with the photos. This is exemplified on Figure 6, which is a screenshot from the video recordings, showing a grandparent and grandchild drawing on top of a photo of the grandparent. The coloured pencils were also used without photos and story books in various ways. They were used for playing games, specifically tic-tac-toe, where the grandparent had one colour, the grandchild another, and the tic-tac-toe board was drawn with a third colour. They were used to write messages, such as "Hello", to the other part. The grandchildren also used them to make drawings for their grandparents (e.g. a rainbow). Playing games together and drawing pictures, either together or for each other, were activities that both participating families reported also being typical of collocated, non-mediated, interaction between the grandparents and their grandchildren.

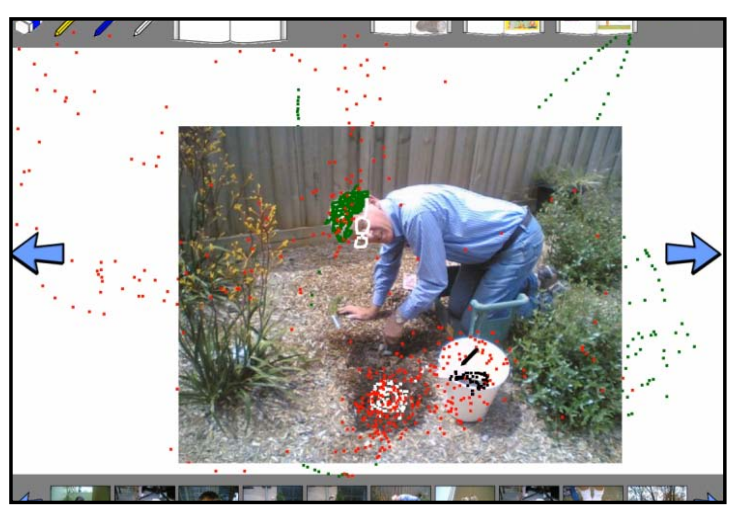

Figure 6: A screenshot from the video recordings, showing a grandfather and granddaughter having a good time together, painting on top of a picture of the grandfather.

\section{Teasing}

Several episodes of mutual teasing were observed in family 2. At one point, the grandparent said: "This is the tale of Peter Rabbit", although the grandchild just moments before had selected another story, which caused the child to answer: "no, it's not" and the grandparent to laugh. Later, the grandparent drew a story character blue, and claimed he was unaware who did it, even though the child suspected the grandparent. The latter is a good example of the grandparent teasing the grandchild in a way that would not be possible in a collocated, non-mediated setting. The grandchildren were just as teasing. Over time a pattern emerged where the grandchild used the white pencil to overwrite, and effectively erasing, the text while the grandparent was reading the story out loud. While erasing, the grandchild often laughed a lot, and it only got funnier when the grandparent started to tease back by erasing the white areas, hence making the text visible again. Another example of mutual teasing involved switching books mid story. While the grandparent was reading out loud, the grandchild suddenly selected another storybook to tease the grandparent.
However, the grandparent chose just to read aloud whatever was shown in front of him, which made the grandchild laugh a lot. This episode of rapidly changing storybooks went on for several minutes.

\section{Bonding}

When the grandparents from family 2 were interviewed after they had used the system, they interestingly reported that the system provided them with opportunities for bonding that would not be possible in a collocated setting. The grandparents agreed that one of their grandchildren were pretty shy when $\mathrm{s}$ /he visited them, but that the same grandchild was "much more free" when s/he used the storytelling system over distance, and that the grandchild probably saw especially the grandfather as more relaxed. This example can be related to previous research, which found that shy individuals fell less inhibited in an online (distributed in space) setting than in an offline (collocated) setting [13].

\section{Interacting with Multiple Grandchildren}

The most common ways the families used the system was one grandparent interacting with one grandchild. However, the video recordings also reveal episodes where one grandchild joined another grandchild in an existing session and episodes where multiple grandchildren participated simultaneously for the entire duration of a session.

The episodes where more than one grandchild interacted simultaneously varied a lot with respect to both the grandchildren's mood and the types of activities conducted. In one session, a grandparent was reading a fictional story to two grandchildren simultaneously, who both answered his questions about the story but otherwise stayed passive and immersed in the reading aloud. In another session, where the grandparent was reading aloud a story, both grandchildren were having a really good time together with the grandparent. They used the pencils to draw on top of story characters, for example, to make the character cry, which made both the grandchildren and the grandparent laugh out loud. At one time it was even observed that one grandchild helped the other grandchild use the system, i.e. selecting a tool. However, just as often, the two grandchildren got annoyed at each other. When one grandchild was being read a story by the grandparent, the other grandchild entered the scene, starting to touch the touch screen. This caused great irritation for the first grandchild, exemplified by loud yelling of "stoooop" or "you're keeping it all to yourself", signalling that one grandchild preferred the other was not touching the touch screen. During the setup in the first family, when the children were given control over the touch screen, not two minutes passed by before one of the children started crying, fighting over control. Interestingly, it only took a few seconds for grandchildren to change their mood during sessions with two simultaneous. One second, the two grandchildren really enjoyed each other's interactions, and the next they were really annoyed at each other. One session had participation of all three grandchildren in the second family, the mother and a grandparent. Two of the grandchildren painted with the pencils, helped by the 
mother, while the third grandchild played the flute for the grandparent. This illustrates the diversity of the system.

\section{Parental Involvement}

The mother in the two families also proved to play an important role for successful use of the probe by the grandchildren. The mother often intervened during active storytelling sessions, and by talking with the grandparents scheduled when the next storytelling session was going to take place. The mother also initiated storytelling sessions and fetched the child or the children only when she had made sure the grandparents were available and had time for a storytelling session. Similarly, she replied to invitations from the grandparent if neither of the children heard the ringing sound and then gathered the children. During sessions, the mother helped the children both to use the system, for example explaining how to enlarge pictures, and suggested activities as "why don't you [the grandparent] read a story to [the grandchild]?". Several times the mother also supplied visual clues to the grandparent about current grandchildren activity. Once, the child did not immediately answer a question from the grandparent and the mother said "she has a cookie in her mouth", thus providing the grandparent with contextual clues from the grandchildren household. Finally, the mother educated her children during sessions, warning one grandchild not to yell into the ear of the other while both were using the system.

\section{Integrating the system into daily life}

The degree at which the two families managed to integrate the system into their daily life was almost as opposite as possible. The first family never managed to integrate it, mostly because the mother did not have the energy to facilitate use. The second family however successfully integrated the system into their daily life to a degree where the children were sad when the probe was recollected from their household. On a side note, this observation in itself presents an interesting ethical concern in relation to longitudinal deployment of technology for research purposes. Figure 7 shows what time of the day the storytelling sessions took place. It shows a clear preference for evening sessions with a secondary preference for early afternoons.

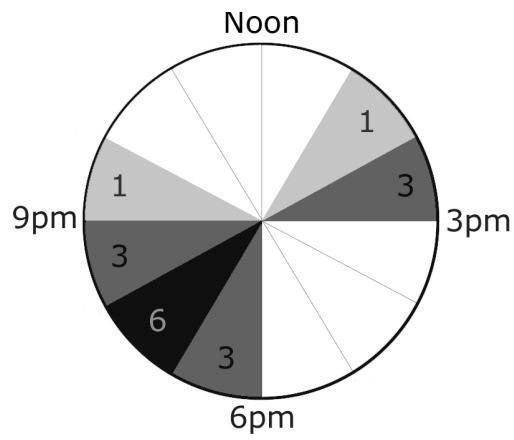

Figure 7: Number and distribution of sessions over time. The darker the colour, the more popular that hour was for storytelling. No sessions were initiated between midnight and noon.
Daily life in the grandchildren's household clearly affected the use of the system. The second family got into a routine where they often conducted storytelling sessions in the evening having the two oldest grandchildren swapping between using the system and having a bath. During sessions, it was also common for the grandchild interacting with the system to be disturbed by other matters in the household. This caused the grandparent to feel insecure if $\mathrm{s} /$ he still had the attention of the grandchild. However, conversely, the use of the system also affected the daily life of the household. As examples, grandchildren would sometimes delay bedtime to have a session with a grandparent, and grandchildren would sometimes disobey the call to dinner because they were interacting with a grandparent.

\section{DISCUSSION}

Based on the findings we derived four themes important for facilitating grandparents and grandchildren communicating synchronously over distance through technology design. Even though these themes are elicited based on empirical data from grandparent-grandchild contact, we hypnotise that the themes are also relevant to consider when designing technology for facilitating contact between distributed parents and children, for example, in cases where one parent travels a lot, or where the parents are divorced.

\section{Conversational Context}

Ballagas et al. found that children have a hard time staying engaged in a phone conversation [2]. Based on a focus group study, Evjemo et al. concluded that parents appreciate communication technologies that provide conversational context [5]. This paper presents an attempt to provide distributed grandparents and grandchildren with conversational context in a real setting. The telling of fictional stories, the ability to share personal photos and the creativity shown with the pencils indicates that both grandchildren and grandparents really enjoyed the storytelling sessions and bonded during so, by sharing the same conversational context. Our study thus supports the need of a conversational context found by Evjemo et al. [5]. Despite the presented technical issues with the system it also appears that it provided better opportunities for bonding than when using just telephones.

\section{Facilitation}

The way the storytelling system was used in the families suggests that it is important to consider the role parents play in facilitating contact between distributed grandparents and grandchildren. Our study shows that parents can greatly increase the amount of bonding contact between the grandparents and the grandchildren. The parents are probably more aware of the routines in the grandparent household than the grandchildren are. This makes them important for prompting the grandchildren to initiate contact at a time of day where there is a higher chance of the grandparents being available. The case of family 1 shows what can happen if neither parent has the time to facilitate use, as both parents were either not home or very busy at home during 
the two weeks this family had the system, resulting in very little use by the grandchildren. During sessions of contact, the parents also play an important role, by supporting the children both technically and conversationally and making sure two concurrently interacting children are not fighting (too much) over control. Thus when designing technology for supporting bonding between distributed grandparents and grandchildren, it is important that the role of the parents is considered as well and that parents are given an opportunity to play a role, as they both can help the children with the technology and have to permit the children to use it.

\section{Diversified interaction forms}

The use of the system in the two families also revealed the importance of diversified interaction forms when designing for children. Children have, similarly to adults, some form of a daily routine. During the day, they're full of energy, running around, playing and are generally just really active. When using the system, similar behaviour was observed with the child interacted wildly, painting fast, and browsing pages fast, thus being active. This is exemplified by one child saying "I'm gonna make you a rainbow" (with the coloured pencils), whereupon the child started painting wildly with all the colours. This is contrasted by behaviour later on the day. The interviews conducted in the two families before the probe was deployed revealed that it was common for the parents to read aloud to the children before they went to bed, or when they were in bed, before they fell asleep. Again, when using the system, similar behaviour could be observed when a child said "Tell me a story" to the grandparents, and then did nothing for almost 20 minutes but turning the pages when instructed to. This suggests that the various ways the children interacted with the system is influenced by the daily routine of the child, who probably expects to be told a story at bedtime whether by the collocated parent or by the remote grandparent. It is also possible that the general mood of the child affects what the child in the moment finds exciting. If the child has had a very energetic day, the child may be interested in a more passive interaction form in the afternoon. No matter if the child was very active or very passive, both the child and the grandparents appreciated the interaction. This suggests that to optimize the possibility for bonding between distributed grandparents and grandchildren to occur, the technology should encompass a diversity of interaction forms to suit the current activity level of the child.

\section{Supporting grandparent caring for grandchild}

When grandparents and grandchildren play together in a collocated setting, the grandparent acts as a carer for play, e.g. by selecting playful artefacts, thus ensuring the child is having fun playing [17]. The grandparent can conduct this behaviour just by watching the child and the environment. Thus, when the grandparents and grandchildren are given the opportunity to be in contact with each other over distance, this role must be attended to, if shared collocated activity is to be mediated successfully. This study showed that the grandparents did indeed conduct the role of caring by asking questions as "do you want me to read you a story?" on the audio channel or dragging personal photos into the shared display, with which the child then played. The video and audio recordings of their interactions also showed that the grandparent adapted his/her activities to the mood of the grandchild. If the grandchild at one point e.g. was not interested in hearing a story, the grandparent immediately suggested other activities. The audio channel alone played an important role in facilitating this, as the grandparent through this got immediate knowledge of the mood of the grandchild, by his/her laughter, voice pitch etc. The grandparent knew s/he had been successful when the child burst out in laughter. These examples show that the grandparent role of caring is important to take into account when designing technologies for improving contact between distributed grandparents and grandchildren. It is also clear that having an audio channel and a shared display provides the grandparent with the possibility to care for the grandchild, thus ensuring the child is having a good time.

\section{Encouraging 'individual play'}

The system also proved to be an instrument of individual play for the grandchildren. This can be illustrated with two types of observed examples. A grandfather was in the process of telling a story to the grandchild, when the grandchild started to interact wildly with the system, switching the pages fast. While the grandfather often just laughed when the child did this, the observed reaction in this episode was different. The grandfather got so annoyed by the grandchild's constant interruptions of his reading that he denied reading anything more that day. A more common observed phenomenon was the grandchild being so immersed in painting with the coloured pencils that questions from the grandparent stayed unanswered despite several attempts by the grandparent to initiate conversation. This suggests that sometimes the grandchild saw the probe more as an instrument of individual play than an instrument of shared activity, which does not work towards more contact between distributed grandparents and grandchildren. This could be addressed by giving the grandparents more control over which tools the grandchildren can use at any time, at the risk, however, of losing the child's interest in the interaction with the grandparent.

\section{Technical issues}

The decision to use loudspeakers and table microphones in both households unfortunately proved to be troublesome as this introduced severe acoustic echo. Speech from one household was played back on the loudspeakers in the other household, and therefore re-recorded and transmitted back again. Especially the grandparents in both families expressed serious irritation over this. The loudspeakers had an important role by playing the telephone sound upon receiving an invitation to participate in a storytelling session to get the attention of the household residents. The table microphones also played an important role as they allowed multiple grandchildren and even multiple parents to chat with the grandparents simultaneously. The microphones 
also captured the general activities in the households, which often functioned as an important primer for conversation. In a future version of the system, acoustic echo cancellation should be applied. Using headsets instead of speakers and table microphones would limit the current walk-up-and-use quality of the system and also limit the peripheral awareness of the remote household created it observably creates.

\section{CONCLUSIONS}

This paper has explored how to design technology to improve contact between grandparents and grandchildren living apart. The design, implementation and deployment of a system in the form of a technology probe have been described, and four important themes have been elicited. The results indicate that grandparents and grandchildren are keen to stay in contact and that this can be facilitated through "conversational context" in the form of joint storytelling involving fictional stories and personal photos. It is also shown that parents play an important role as facilitators of contact between grandchildren and grandparents. The daily rhythms, and how this affects the activity level of the child, must also be given thought when designing technology to be used partly by children. The study also shows the importance of taking the grandparent's role of carer for play into consideration to allow meaningful and fun interactions to arise. It is hoped these themes will help fellow researchers when designing technology for improving contact between grandparents and grandchildren living apart.

\section{LIMITATIONS}

During analysis, it was impossible to be absolutely sure of who was interacting with the system when a storytelling session was in progress. This is because several members of the same household often used the system concurrently and because the probe did not record visual information about what was happening in front of the display. The current user interacting with the system was therefore often determined by an educated guess based on the audio channel. Furthermore, the generalisability of the presented findings and themes needs to be examined through further studies. Use patterns might be different in other families, potentially leading to discovery of more themes.

\section{FUTURE WORK}

As the study revealed a preference to use the system in the evenings, it is expected that grandparents and grandchildren face further challenges for creating and maintaining a relationship if living in different time zones. This scenario is currently being studied through a deployment of the Storytelling system at two families with grandparents and grandchildren distributed between Denmark and Australia. Findings from this study will be reported in a future paper.

\section{REFERENCES}

1. Ames, M. G., Go, J., Kaye, J. ' and Spasojevic, M. (2010). Making love in the network closet: the benefits and work of family videochat. Proc. CSCW'10, ACM, 145-154.
2. Ballagas, R., Kaye, J., Ames, M., Go, J. and Raffle, H. (2009). Family communication: phone conversations with children. Proc. IDC'09. ACM, 321-324.

3. Bos, N., Olson, J., Gergle, D., Olson, G., and Wright, Z. (2002). Effects of four computer-mediated communications channels on trust development. Proc. CHI'02. ACM, 135-140.

4. CamStudio. http://www.camstudio.org

5. Evjemo, B., Svendsen, G. B., Rinde, E., and Johnsen, J. K. (2004). Supporting the distributed family: the need for a conversational context. Proc. NordiCHI'04, ACM, 309-312.

6. Hutchinson, H., Mackay, W., et al. (2003). Technology probes: inspiring design for and with families. Proc. CHI'03. ACM, NY, 17-24.

7. Kennedy, G. E. (1992). Shared activities of grandparents and grandchildren. In Psychol Rep. 1992, Feb; 70(1): 211-27.

8. Kornhaber, A. (1996). Contemporary Grandparenting. Thousand Oaks, CA: Sage Publications, Inc.

9. Kornhaber, A., Woodward, K. L. (1981). The grandparents/grandchildren. The vital connection. Garden City, NY: Anchor Press/Doubleday.

10. Kraut, R. E., Gergle, D., and Fussell, S. R. (2002). The use of visual information in shared visual spaces: informing the development of virtual co-presence. Proc. CSCW'02. ACM, 31-40.

11. Modlitba, P. L. (2008). GlobeToddler: enhancing the experience of remote interaction for preschool children and their travelling parents. Master's Thesis. MIT Media Laboratory. Http://web.media.mit.edu/ paulina/ portfolio/PaulinaModlitbaMSThesis.pdf.

12. Raffle, H., Ballagas, R., Revelle, G., Horii et al. (2010). Family story play: reading with young children (and elmo) over a distance. Proc. CHI '10. ACM, 1583-1592.

13. Roberts et al. (2000). "U r a lot bolder on the net": Shyness and internet use. In: Crozier, W.R. (Ed.) Shyness: Development, consolidation and change, Routledge, New York, 121-138.

14. Stefik, M., Bobrow, D. G., Foster, G., Lanning, S., and Tatar, D. (1987). WYSIWIS revised: early experiences with multiuser interfaces. ACM Trans. Inf. Syst. 58(2): 147-167.

15. Strauss, A and Corbin, J. (1990). Basics of Qualitative Research, Newbury Park: Sage.

16. Ventrilo. http://www.ventrilo.com.

17. Vetere, F., Davis, H., Gibbs, M. et al. (2009). The Magic Box and Collage: Responding to the challenge of distributed intergenerational play. Int. J. Hum.-Comput. Stud. 67(2): 165-178.

18. Williams, B. K., Sawyer, S. C. and Wahlstrom, C. M. (2005). Marriages, Families \& Intimate Relationships. Boston, MA: Pearson.

19. Yarosh, S., Cuzzort, S., Müller, H. and Abowd, G. D. (2009). Developing a media space for remote synchronous parent-child interaction. Proc. IDC'09, ACM, $97-$ 105. 